\title{
Dust, Ice, and Gas In Time (DIGIT) Herschel` program first results
}

\section{A full PACS-SED scan of the gas line emission in protostar DK Chamaeleontis}

\author{
T. A. van Kempen ${ }^{1}$, J. D. Green ${ }^{2}$, N. J. Evans $\mathrm{II}^{2}$, E. F. van Dishoeck ${ }^{3,4}$, L. E. Kristensen ${ }^{3}$, G. J. Herczeg ${ }^{4}$, B. Merín ${ }^{5}$, \\ J.-E. Lee ${ }^{6}$, J. K. Jørgensen ${ }^{7}$, J. Bouwman ${ }^{8}$, B. Acke ${ }^{9,24}$, M. Adamkovics ${ }^{10}$, J. C. Augereau ${ }^{11}$, E. Bergin ${ }^{12}$, \\ G. A. Blake ${ }^{13}$, J. M. Brown ${ }^{4}$, J. S. Carr ${ }^{14}$, J.-H. Chen ${ }^{2}$, L. Cieza ${ }^{15}$, C. Dominik ${ }^{16,17}$, C. P. Dullemond ${ }^{8,18}$,
} M. M. Dunham ${ }^{2}$, A. Glassgold ${ }^{10}$, M. Güdel ${ }^{19}$, P. M. Harvey ${ }^{2}$, Th. Henning ${ }^{8}$, M. R. Hogerheijde ${ }^{3}$, D. Jaffe ${ }^{2}$, H. J. Kim² ${ }^{2}$, C. $\mathrm{Knez}^{20}$, J. H. Lacy ${ }^{2}$, S. Maret ${ }^{11}$, G. Meeus ${ }^{21}$, R. Meijerink ${ }^{3}$, G. D. Mulders ${ }^{16,22}$, L. Mundy ${ }^{20}$, J. Najita ${ }^{23}$, J. Olofsson ${ }^{8}$, K. M. Pontoppidan ${ }^{13}$, C. Salyk ${ }^{2}$, B. Sturm ${ }^{8}$, R. Visser ${ }^{3}$, L. B. F. M. Waters ${ }^{9,16}$,

C. Waelkens ${ }^{9}$, and U. A. Yildiz ${ }^{3}$

(Affiliations are available in the online edition)

Received 31 March 2010 / Accepted 14 May 2010

\begin{abstract}
Aims. We aim to study the composition and energetics of the circumstellar material of DK Cha, an intermediate-mass star in transition from an embedded configuration to a star plus disk stage, during this pivotal stage of its evolution.

Methods. Using the range scan mode of PACS on the Herschel Space Observatory, we obtained a spectrum of DK Cha from 55 to $210 \mu \mathrm{m}$ as part of the DIGIT key program.

Results. Almost 50 molecular and atomic lines were detected, many more than the 7 lines detected in ISO-LWS. Nearly the entire ladder of CO from $J=14-13$ to $38-37\left(E_{u} / k=4080 \mathrm{~K}\right)$, water from levels as excited as $J_{K_{-1} K_{+1}}=7_{07}\left(E_{u} / k=843 \mathrm{~K}\right)$, and $\mathrm{OH}$ lines up to $E_{u} / k=290 \mathrm{~K}$ were detected.

Conclusions. The continuum emission in our PACS SED scan matches the flux expected by a model consisting of a star, a surrounding disk of $0.03 M_{\odot}$, and an envelope of a similar mass, supporting the suggestion that the object is emerging from its main accretion stage. Molecular, atomic, and ionic emission lines in the far-infrared reveal the outflow's influence on the envelope. The inferred hot gas may be photon-heated, but some emission may be caused by $\mathrm{C}$-shocks in the walls of the outflow cavity.
\end{abstract}

Key words. astrochemistry - circumstellar matter - stars: formation - stars: protostars - infrared: ISM - submillimeter: ISM

\section{Introduction}

One of the pivotal points in the formation of a star occurs when the star and disk system emerges from the collapsing envelope, signaling the end of the main accretion of stellar mass. The transition from a stage I protostar, embedded in a substantial envelope, to a stage II configuration, with only a star and $\operatorname{disk}^{1}$, is poorly understood.

At a distance of $178 \pm 18$ pc IRAS $12496-7650$ is the brightest infrared (IR) and submillimeter (submm) source in the Chamaeleon II star-forming region, (Whittet et al. 1997). Follow-up studies to the discovery of IRAS $12496-7650$ by Hughes et al. (1989, 1991), along with subsequent observations (e.g., Whittet et al. 1997; Young et al. 2005; Porras et al. 2007; Spezzi et al. 2008), identified the variable Herbig Ae star DK Cha as the optical counterpart. The central star mass is estimated to be 2-3 $M_{\odot}$ (Spezzi et al. 2008) with a bolometric luminosity between 24 (Spezzi et al. 2008) and $35 L_{\odot}$ (van Kempen et al. 2009a). The star is surrounded by a molecular outflow and

${ }^{\star}$ Herschel is an ESA space observatory with science instruments provided by the European-led Principal Investigator consortia and with important participation from NASA.

${ }^{1}$ See, e.g., Robitaille et al. (2007) for a definition of the stages. protostellar envelope (van Kempen et al. 2006; van Kempen et al. 2009a). The spectral energy distribution (SED) is roughly flat with wavelength from the near-infrared to mid-infrared (Hughes et al. 1989). Using the $T_{\text {bol }}$ definition and including longer wavelength data (e.g., Henning et al. 1993; van Kempen et al. 2009a), DK Cha has $T_{\text {bol }}=580 \mathrm{~K}$, making it a class I source, but near the class II boundary at $650 \mathrm{~K}$. Owing to an approximately face-on viewing angle down the outflow cone (van Kempen et al. 2009a), one can observe the protostellar disk directly, unobstructed by the surrounding envelope. This viewing angle also allows the optical emission to escape. The observational SED classes are often assumed to correspond to the physical stages, but this is by no means obvious when aspherical models are examined (e.g., Robitaille et al. 2007; Crapsi et al. 2008; Dunham et al. 2010) or tracers of dense gas are considered (van Kempen et al. 2009b). The same physical object can have different SED classifications depending on the viewing angle. Thus, it is interesting to study an object that is near an SED classification boundary and that also appears to be transitional in physical stage.

Strong far-IR continuum and line emission have been detected (Giannini et al. 1999; van Kempen et al. 2006). As discussed by van Dishoeck (2004); Nisini et al. (2005), and 
van Kempen et al. (2010) for the similar source HH46, far-IR atomic, ionic, and molecular lines can constrain the properties of the disk, envelope, and outflow. The $\mathrm{CO}$ emission lines between $J=14-13$ and $J=19-18$, as well as [O I] and [C II], were observed from DK Cha with ISO-LWS, but other possible molecular species, such as $\mathrm{OH}$ and $\mathrm{H}_{2} \mathrm{O}$, were undetected (Lorenzetti et al. 1999; Giannini et al. 1999).

The large beam of ISO-LWS (90") and its low spectral resolving power $(\lambda / \Delta \lambda=200)$ were insufficient to determine the origin (e.g., envelope versus outflow) of the $\mathrm{CO}$ emission. The preferred picture was of a small, quiescent hot core of 300 AU around the protostar (Giannini et al. 1999), but observations of $\mathrm{CO} 4-3$ and 7-6 indicate that this warm $\mathrm{CO}$ emission either originates outside the inner few thousand $\mathrm{AU}$ or cannot be heated simply by radiation from the central star (passive heating) (van Kempen et al. 2006); the far-IR and submm lines have different energies and critical densities, so they may originate in different regions.

The Herschel key programme Dust, Ice, and Gas In Time (DIGIT) aims to study the evolution of these three constituents through the star formation process by observing objects at different stages. This paper, along with a companion paper on HD100546, a stage II object (Sturm et al. 2010), present the first results from the DIGIT science demonstration observations.

\section{Observations}

The launch of the Herschel Space Observatory (Pilbratt 2010) with the Photodetector Array Camera and Spectrometer (PACS) instrument (Poglitsch 2010) oboard, enables the acquisition of far-IR spectra with high spectral resolving power and good spatial $\left(\sim 9^{\prime \prime}\right)$ resolution. The range scan/SED mode provides a full spectrum $(55-210 \mu \mathrm{m})$.

DK Cha was observed for a total of $\sim 4 \mathrm{~h}$ on 10 December 2009 by the PACS spectrometer on the Herschel Space Observatory in pointed range-scan spectroscopy mode (obsid 1342188039 and 1342188040$)$. An image slicer rearranges the light within a $5 \times 5$ array, with each spatial pixel (spaxel) covering 9.'4 $\times$ 9.'4. A grating disperses the light onto two $16 \times 25$ pixel Ge:Ga detectors. Observations were taken using nodding, in which two off-positions separated by \pm 6 arcmin in elevation were taken as "blank" sky, from which the signal is subtracted. Consequently, spatially diffuse lines (e.g., [C II]) are subtracted out.

The effective spectral resolving power was about $1000+/-500$, lower than expected, because of the pointing error, which is perpendicular to the slit, and the effects of fringing on the continuum. The point-spread function of PACS increases towards longer wavelengths and is larger than a single spaxel, but is corrected in post-processing using the PACS spectrum of the point-like HD100546 (Sturm et al. 2010) to calculate the extraction efficiencies.

The data were reduced using the Herschel interactive processing environment (HIPE v. 2.4). The observation was slightly mispointed $\left(\sim 5-6^{\prime \prime}\right)$ making it difficult to assess whether the emission is extended and causes wavelength shifts in some spectral ranges. Because of the mispointing, uncertainty in the spectral response function, light leakage problems, and uncertainties in the wavelength dependence of the PSF, the spectrum was trimmed shortward of $56 \mu \mathrm{m}$, longward of $190 \mu \mathrm{m}$, and in the range 95-101 $\mu \mathrm{m}$. Two spectra were used to identify lines: One from the central spaxel alone and a second spectrum, which was used for most lines, craeted by summing the fluxes from all spatial pixels to correct for the PSF and mispointing. We focus on gas lines in this Letter.
Table 1. Detected gas lines within the PACS spectrum of DK Cha.

\begin{tabular}{|c|c|c|c|c|c|}
\hline Species & Transition & $\begin{array}{c}\lambda^{a} \\
\mu \mathrm{m}\end{array}$ & $\begin{array}{r}E_{u} \\
K\end{array}$ & $\begin{array}{r}F W H M \\
\mu \mathrm{m}\end{array}$ & $\begin{array}{r}\text { Flux }^{b} \\
\mathrm{~W} \mathrm{~m}^{-2}\end{array}$ \\
\hline$[\mathrm{OI}]$ & ${ }^{3} \mathrm{P}_{1-{ }^{-}}{ }^{\mathrm{P}_{2}}$ & 63.18 & 227.72 & 0.08 & 3657.7 \\
\hline $\mathrm{OH} 3 / 2-3 / 2$ & $9 / 2--7 / 2+$ & 65.13 & 512.1 & 0.05 & $56.0^{2}$ \\
\hline $\mathrm{OH} 3 / 2-3 / 2$ & $9 / 2+-7 / 2-$ & 65.28 & 510.9 & 0.07 & $52.3^{2}$ \\
\hline $\mathrm{CO}$ & $38-37$ & 69.07 & 4079.98 & 0.06 & $42.1^{2}$ \\
\hline $\mathrm{CO}$ & $37-36$ & 70.91 & 3871.88 & 0.09 & $59.6^{2}$ \\
\hline $\mathrm{o}-\mathrm{H}_{2} \mathrm{O}^{3}$ & $7_{07}-6_{16}$ & 71.95 & 843.5 & 0.06 & $37.1^{2}$ \\
\hline $\mathrm{CO}$ & $36-35$ & 72.84 & 3668.96 & 0.06 & $40.1^{2}$ \\
\hline $\mathrm{CO}$ & $35-34$ & 74.89 & 3471.44 & 0.1 & $57.0^{2}$ \\
\hline $\mathrm{o}-\mathrm{H}_{2} \mathrm{O}$ & $4_{23}-3_{12}$ & 78.74 & 432.2 & 0.04 & $19.8^{2}$ \\
\hline $\mathrm{OH} 1 / 2-3 / 2$ & $1 / 2--3 / 2+$ & 79.12 & 181.7 & 0.06 & $28^{2}$ \\
\hline $\mathrm{CO}$ & $33-32$ & 79.36 & 3092.45 & 0.03 & $17.5^{2}$ \\
\hline $\mathrm{CO}$ & $32-31$ & 81.81 & 2911.15 & 0.05 & 126.2 \\
\hline $\mathrm{o}-\mathrm{H}_{2} \mathrm{O}$ & $6_{16}-5_{05}$ & 82.09 & 643.5 & 0.03 & 96.2 \\
\hline $\mathrm{CO}$ & $31-30$ & 84.41 & 2735.28 & 0.09 & 336.0 \\
\hline $\mathrm{OH} 3 / 2-3 / 2$ & $7 / 2+-5 / 2-$ & $84.42^{1}$ & 291.2 & - & 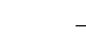 \\
\hline $\mathrm{OH} 3 / 2-3 / 2$ & $7 / 2--5 / 2+$ & 84.60 & 290.5 & 0.09 & 301.4 \\
\hline $\mathrm{CO}$ & $30-29$ & 87.19 & 2564.94 & 0.09 & 317.5 \\
\hline $\mathrm{CO}$ & $29-28$ & 90.16 & 2399.93 & 0.14 & 545.8 \\
\hline $\mathrm{CO}$ & $28-27$ & 93.35 & 2240.30 & 0.07 & 261.4 \\
\hline $\mathrm{CO}$ & $27-26$ & 96.77 & 2086.22 & 0.10 & 330.1 \\
\hline $\mathrm{CO}$ & $25-24$ & 104.44 & 1794.23 & 0.2 & 314.5 \\
\hline $\mathrm{o}-\mathrm{H}_{2} \mathrm{O}$ & $2_{21}-1_{10}$ & 108.08 & 194.1 & 0.15 & 126.9 \\
\hline $\mathrm{CO}$ & $24-23$ & 108.76 & 1656.55 & 0.15 & 260.0 \\
\hline $\mathrm{CO}$ & $23-22$ & 113.46 & 1524.19 & 0.2 & 477.4 \\
\hline $\mathrm{o}-\mathrm{H}_{2} \mathrm{O}$ & $4_{14}-3_{03}$ & $113.54^{1}$ & 323.5 & - & - \\
\hline $\mathrm{CO}$ & $22-21$ & 118.58 & 1397.43 & 0.17 & 285.2 \\
\hline $\mathrm{OH} 3 / 2-3 / 2$ & $5 / 2--3 / 2+$ & 119.23 & 120.7 & 0.18 & $<40$ \\
\hline $\mathrm{OH} 3 / 2-3 / 2$ & $5 / 2+-3 / 2-$ & 119.44 & 120.5 & 0.17 & $<72.3$ \\
\hline$[\mathrm{N}$ II $]$ & ${ }^{3} \mathrm{P}_{2}-{ }^{3} \mathrm{P}_{1}$ & 121.90 & 188.20 & 0.12 & 45.7 \\
\hline $\mathrm{CO}$ & $21-20$ & 124.19 & 1276.10 & 0.17 & 357.1 \\
\hline $\mathrm{p}-\mathrm{H}_{2} \mathrm{O}$ & $4_{04}-3_{13}$ & 125.35 & 319.5 & 0.12 & 42.8 \\
\hline $\mathrm{CO}$ & $20-19$ & 130.37 & 1160.24 & 0.16 & 319.0 \\
\hline $\mathrm{CO}$ & 19-18 & 137.20 & 1049.89 & 0.18 & 338.7 \\
\hline $\mathrm{p}-\mathrm{H}_{2} \mathrm{O}$ & $3_{13}-2_{02}$ & 138.53 & 204.7 & 0.17 & 103.0 \\
\hline $\mathrm{CO}$ & $18-17$ & 144.78 & 945.01 & 0.15 & 350.4 \\
\hline$[\mathrm{OI}]$ & ${ }^{3} \mathrm{P}_{0}-{ }^{3} \mathrm{P}_{2}$ & 145.53 & 326.58 & 0.16 & 146.7 \\
\hline $\mathrm{CO}$ & $17-16$ & 153.27 & 845.63 & 0.15 & 386.0 \\
\hline $\mathrm{CO}$ & $16-15$ & 162.81 & 751.76 & 0.18 & 466.5 \\
\hline $\mathrm{OH} 1 / 2-1 / 2$ & $3 / 2+-1 / 2-$ & 163.12 & 270.2 & 0.24 & 91.0 \\
\hline $\mathrm{OH} 1 / 2-1 / 2$ & $3 / 2+-1 / 2$ & 163.40 & 269.8 & 0.19 & 90.0 \\
\hline $\mathrm{CO}$ & $15-14$ & 173.63 & 663.37 & 0.16 & 413.0 \\
\hline $\mathrm{o}-\mathrm{H}_{2} \mathrm{O}$ & $3_{03}-212$ & 174.63 & 196.8 & 0.19 & 208.0 \\
\hline $\mathrm{o}-\mathrm{H}_{2} \mathrm{O}$ & $2_{12}-1_{01}$ & 179.53 & 114.4 & 0.11 & 31.0 \\
\hline $\mathrm{o}-\mathrm{H}_{2} \mathrm{O}$ & $2_{21}-2_{12}$ & 180.49 & 194.1 & 0.12 & 34.9 \\
\hline $\mathrm{CO}$ & $14-13$ & 186.00 & 580.51 & 0.20 & 437.2 \\
\hline
\end{tabular}

Notes. ${ }^{(a)}$ Laboratory wavelength; ${ }^{(b)}$ flux summed over all pixels in $10^{-18} \mathrm{~W} \mathrm{~m}^{-2}$. Typical line flux uncertainties are dominated by calibration uncertainties $(\sim 30-50 \%)$ and fringing $(0.1 \mathrm{Jy}$ at the longest wavelengths to $0.6 \mathrm{Jy}$ at the shortest); (1) lines blended with above line; (2) Line detected only in central spaxel and should not be compared to the fluxes of the summed spaxels; ${ }^{(3)}$ doublet unresolved.

\section{Results}

\subsection{Continuum}

The total SED in Fig. 1 includes photometry from the literature (Alcalá et al. 2008; Spezzi et al. 2008; Evans et al. 2009, Nefs et al., in prep), combined with Spitzer-IRS spectroscopy and the PACS results. The photometric points were fitted by the SED fitter routine from Robitaille et al. (2007), the resultin parameters being shown in Table 2. Because of the the extinction and variability at short wavelengths, only points longwards of $3 \mu \mathrm{m}$ were considered as input to the fit. The best-fit model is overplotted and divided into the contributions from the star, disk, and envelope. The short-wavelength IRS continuum is dominated by the flux from the disk, the longer wavelength IRS continuum covers 


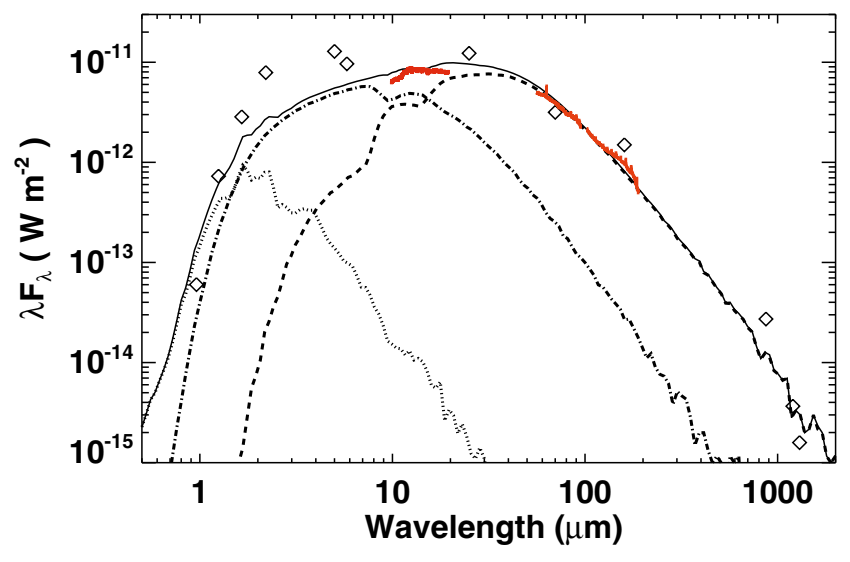

Fig. 1. SED of DK Cha using WFI $I$-band data, 2 MASS $(J, H, K)$ and Spitzer-IRAC (3.6 and $4.5 \mu \mathrm{m}$. The other bands, 5.8 and $8.0 \mu \mathrm{m}$ not being shown due to saturation), Spitzer-MIPS (24, 70 and $160 \mu \mathrm{m})$, APEX-Laboca $(870 \mu \mathrm{m})$ and SEST $(1.2$ and $1.3 \mathrm{~mm})$. Overplotted are the best-fit model from the Robitaille et al. (2007) grid and the PACS and Spitzer-IRS spectra. The MIPS $70 \mu \mathrm{m}$ is corrected for saturation; the $160 \mu \mathrm{m}$ for large-scale emission. The solid line is the full model, while the dashed line shows the envelope contribution, the dotdashed the disk contribution, and the dotted that of the star. Photometric points are shown as diamonds. The short-wavelength points are not well matched. Fluxes at short wavelength $(<3 \mu \mathrm{m})$ are variable.

Table 2. Best-fit from Robitaille models.

\begin{tabular}{ll|ll}
\hline \hline \multicolumn{4}{c}{ DK Cha characteristics } \\
\hline Envelope mass & $0.03 M_{\odot}$ & $A_{V}{ }^{1}$ & 7.9 \\
Disk mass & $0.03 M_{\odot}$ & Inclination & $<18^{\circ}$ \\
Luminosity & $29.4 L_{\odot}$ & Disk Inner Radius & $0.3 \mathrm{AU}$ \\
\hline
\end{tabular}

Notes. ${ }^{(1)}$ Total $A_{\mathrm{v}}$ of which $A_{\mathrm{v}}=5.2 \mathrm{mag}$. is attributed to the envelope at an inclination of $18^{\circ}$ and 2.7 mag. to more extended cloud.

the transition from disk to envelope, and the PACS continuum arises in the envelope. The model underestimates the observations in the near-IR and mid-IR, indicating that the disk emission is underestimated in the model.

The best-fit models of the SED grid indeed confirm the assumption of a nearly face-on configuration $<18^{\circ}$ with relatively low and equal envelope and disk masses of $0.03 M_{\odot}$, indicative of a source in transition from stage I to stage II (Robitaille et al. 2007). The PACS continuum was not used to constrain the fit, the consistency with the predicted model (differences of $<10 \%$ ) thus being encouraging, especially considering the calibration uncertainty of $30-50 \%$.

\subsection{Line inventory}

Table 1 lists all gas lines that have been identified. Figure 2 shows the spectrum after subtraction of a polynomial fit to the continuum. We used a combination of the ISO line list ${ }^{2}$ and the list published in Lerate et al. (2006) for the Orion bar. More lines may be detectable in this spectrum after PSF deconvolution. Some lines in Table 1 are only detected in the central spaxel. Owing to the fringing in other spaxels at short wavelengths and the PSF problems discussed above, these lines cannot be seen in the summed result of Fig. 2. Using spectra from both the summation of all 25 spaxels and from only the central spaxel, a large range of $\mathrm{CO}, \mathrm{H}_{2} \mathrm{O}$, and $\mathrm{OH}$ lines were detected, ranging in

\footnotetext{
${ }^{2}$ http://www.mpe-garching.mpg.de/iso/linelists/ Molecular.html
}

excitation energies from a few hundred kelvin to over $3000 \mathrm{~K}$. In addition, [O I] and [N II] were identified. Emission from [C II] at $157.7 \mu \mathrm{m}$ is not seen. That line was detected by Lorenzetti et al. (1999), but was interpreted to be extended emission, associated with the galactic ISM. This component is subtracted out in our observations due to the nodding, which was not used in ISO-LWS.

\subsection{Gas analysis}

infrared CO lines were previously detected by Giannini et al. (1999), but only up to CO $J=19-18$. Assuming the lines to be optically thin, those could be fitted with temperatures ranging from $T=200 \mathrm{~K}$ to $T=750 \mathrm{~K}$ and densities from $n=4 \times 10^{6} \mathrm{~cm}^{-3}$ to $n=5 \times 10^{5} \mathrm{~cm}^{-3}$. The high $T$, low $n$ model, which produces the most emission in the high- $J$ lines, is overplotted in Fig. 3. As can be seen, this model severely underpreditcs the intensity of the $\mathrm{CO}$ lines from higher transitions seen by Herschel. The other proposed models predict even less high- $J$ emission.

The CO level populations up to $J=31$ (Fig. 3), can be fitted with two distinct rotational temperatures $\left(T_{\text {Rot }}\right)$, a break occurring at about $E_{\text {up }}=1500 \mathrm{~K}$. Most of the mid-J CO lines can be fitted by $T_{\text {Rot }} \sim 380 \mathrm{~K}$, while higher excitation lines are best fitted with $T_{\text {Rot }} \sim 1365 \mathrm{~K}$. In HH46 (van Kempen et al. 2010), UV photon heating (Spaans et al. 1995) predominates for lines with energies below $1700 \mathrm{~K}$, while a non-dissociative C-shock has a more important effect than the emission from higher excitation lines. A similar model is plausible for DK Cha, but this issue will be explored in a forthcoming paper (Green et al. in prep).

Water was detected in a wide variety of transitions, up to $7_{07}-$ $6_{16}$ at $71.93 \mu \mathrm{m}$ with $(E / k=843 \mathrm{~K})$. Water was not detected in ISO-LWS (Giannini et al. 1999) because the lines are 5 times weaker than the CO lines. The discovery of the $7_{07}-6_{16}$ line is indicative of high temperature, as confirmed by the high- $J \mathrm{CO}$ lines. Given the possible identification of shocked CO and the geometry of DK Cha, it is likely that the water emission arises at least in part from either a non-dissociative C-shock or UVheating of the quiescent material along the outflow cavity walls.

In addition, at least 9 lines of $\mathrm{OH}$ are detected. Since oxygen is rapidly transformed into $\mathrm{H}_{2} \mathrm{O}$ at temperatures higher than $100 \mathrm{~K}$ and frozen out below that, either a very strong UV field or a dissociative shock is needed. $\mathrm{OH}$ is a major coolant for dissociative shocks (Neufeld \& Dalgarno 1989). This dissociative shock is accompanied by a C-shock, which may explain the $\mathrm{CO}$ and $\mathrm{H}_{2} \mathrm{O}$ analysis. Models from Wampfler et al. (in prep) also suggest that $\mathrm{C}$-shock densities are often too low to account for both these high excitation lines and at the same time not overproduce the 119 and $163 \mu \mathrm{m} \mathrm{OH}$ lines.

HD 100546 (Sturm et al. 2010), a Herbig Be star without an envelope, was chosen for early observations to be a source analogous to DK Cha, but more evolved. A comparison is useful because similar species are seen in its spectrum. Emission of $\mathrm{H}_{2} \mathrm{O}$ and $\mathrm{OH}$ from the upper layers of a flared, but quiescent disk, similar to that around HD 100546 (Sturm et al. 2010), may account for some of the lines from DK Cha. However, accounting for the difference in distance, the CO lines from DK Cha are more than 10 times stronger, and higher excitation lines from $\mathrm{H}_{2} \mathrm{O}$ are seen toward DK Cha.

A possible explanation of the stronger emission of DK Cha is an accretion shock onto the disk, where high densities and temperatures are present. Another possibility is a shock in the walls of the outflow, which will also be examined further in Green et al. (in prep.). 


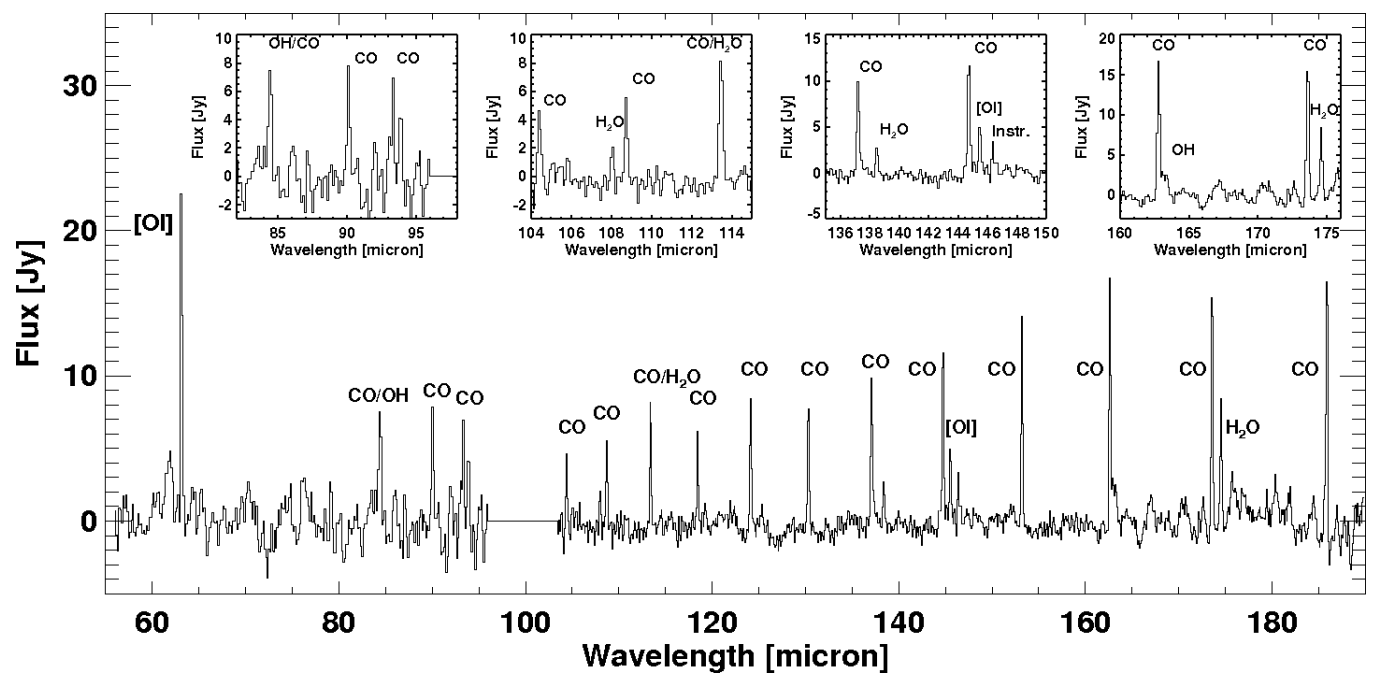

Fig. 2. The spectrum from 55-190 $\mu \mathrm{m}$ of all spaxels added together, after removal of the continuum and rebinning to $0.5 \mu \mathrm{m}$ resolution. Several dips or bumps (e.g., around $75 \mu \mathrm{m}$ ) are instrument artifacts. Inserts show expanded views of parts of the spectrum, with line identifications.
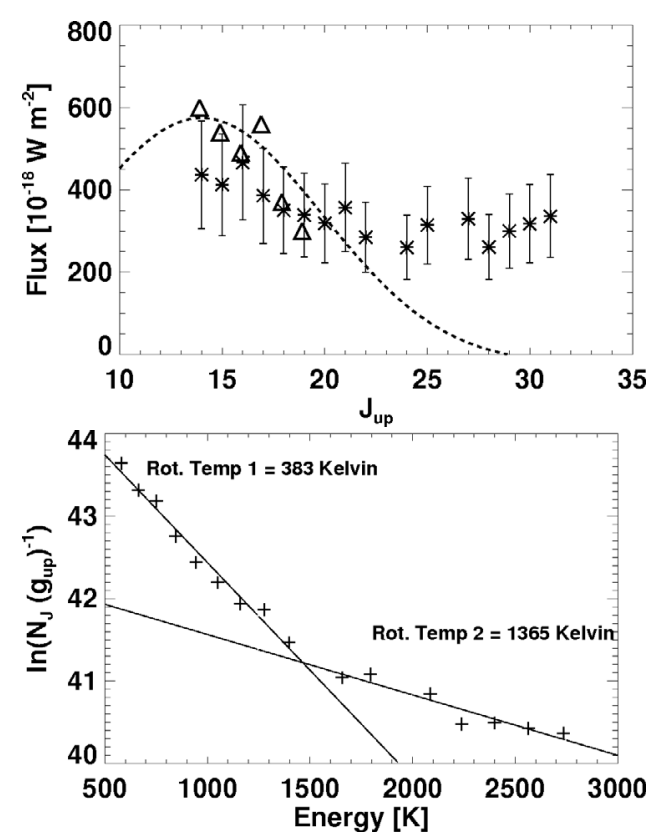

Fig. 3. Upper: CO line fluxes plotted versus $J_{\text {up. The ISO line fluxes }}$ are shown with triangles. A clear excess can be seen in the CO line fluxes from $J_{\text {up }}=20$ onwards compared to the warmest model of $750 \mathrm{~K}$ proposed by Giannini et al. (1999) (dashed line). Lower: CO Rotational diagram, showing two distinct populations with a break around $1500 \mathrm{~K}$ in energy. The blended lines are excluded from the fit and the plot. CO lines with $J_{\text {up }}$ larger than 31 are excluded from both plots, as these lines are detected only in the central spaxel.

\section{Conclusions}

We have found that the continuum models that fit the data of DK Cha are consistent with the interpretation that it is a transitional obsject between a stage I and stage II, with nearly equal masses in the envelope and disk and a nearly face-on geometry. The full PACS SED mode observations reveal an extremely rich spectrum of over 50 ionic, atomic, and molecular lines. With additional improvements to the spectral response function, we will be able to identify additional gas, ice, and dust features in the spectrum. The full range scan/SED mode will be able to probe many aspects of a protostellar object, from the ambient envelope to gas excited by ultraviolet radiation and by shocks.

Acknowledgements. Support for this work, part of the Herschel Open Time Key Project Program, was provided by NASA through an award issued by the Jet Propulsion Laboratory, California Institute of Technology. The authors would like to thank Bart VandenBussche and Alessandra Contursi for help with the data reduction, and David Ardila for his help in scheduling the observations prior to the ESAC SDP workshop.

\section{References}

Alcalá, J. M., Spezzi, L., Chapman, N., et al. 2008, ApJ, 676, 427

Crapsi, A., van Dishoeck, E. F., Hogerheijde, M. R., Pontoppidan, K. M., \& Dullemond, C. P. 2008, A\&A, 486, 245

Dunham, M. M., Evans, N. J., Terebey, S., Dullemond, C. P., \& Young, C. H. 2010, ApJ, 710, 470

Evans, N. J., Dunham, M. M., Jørgensen, J. K., et al. 2009, ApJS, 181, 321

Giannini, T., Lorenzetti, D., Tommasi, E., et al. 1999, A\&A, 346, 617

Henning, T., Pfau, W., Zinnecker, H., \& Prusti, T. 1993, A\&A, 276, 129

Hughes, J. D., Emerson, J. P., Zinnecker, H., \& Whitelock, P. A. 1989, MNRAS, 236, 117

Hughes, J. D., Hartigan, P., Graham, J. A., Emerson, J. P., \& Marang, F. 1991, AJ, 101, 1013

Lerate, M. R., Barlow, M. J., Swinyard, B. M., et al. 2006, MNRAS, 370, 597

Lorenzetti, D., Tommasi, E., Giannini, T., et al. 1999, A\&A, 346, 604

Neufeld, D. A., \& Dalgarno, A. 1989, ApJ, 340, 869

Nisini, B., Antoniucci, S., Giannini, T., \& Lorenzetti, D. 2005, A\&A, 429, 543

Pilbratt, G. L., Riedinger, J. L., Passvogel, T., et al. 2010, A\&A, 518, L1

Poglitsch, A., Waelkens, C., Geis, N., et al. 2010, A\&A, 518, L2

Porras, A., Jørgensen, J. K., Allen, L. E., et al. 2007, ApJ, 656, 493

Robitaille, T. P., Whitney, B. A., Indebetouw, R., \& Wood, K. 2007, ApJS, 169, 328

Spaans, M., Hogerheijde, M. R., Mundy, L. G., \& van Dishoeck, E. F. 1995, ApJ, 455, L167

Spezzi, L., Alcalá, J. M., Covino, E., et al. 2008, ApJ, 680, 1295

Sturm, B., Bouwman, J., Henning, Th. et al. 2010, A\&A, 518, L129

van Dishoeck, E. F. 2004, ARA\&A, 42, 119

van Kempen, T. A., Hogerheijde, M. R., van Dishoeck, E. F., et al. 2006, A\&A, 454, L75

van Kempen, T. A., van Dishoeck, E. F., Hogerheijde, M. R., \& Güsten, R. 2009a, A\&A, 508, 259

van Kempen, T. A., van Dishoeck, E. F., Salter, D. M., et al. 2009b, A\&A, 498, 167

van Kempen, T. A., Kristensen, L. E, Herzceg, G. J, et al. 2010, A\&A, 518, L121 Whittet, D. C. B., Prusti, T., Franco, G. A. P., et al. 1997, A\&A, 327, 1194

Young, K. E., Harvey, P. M., Brooke, T. Y., et al. 2005, ApJ, 628, 283 
1 Harvard-Smithsonian Center for Astrophysics, 60 Garden Street, MS 78, Cambridge, MA 02138, USA

e-mail: tvankempen@cfa.harvard.edu

2 The University of Texas at Austin, Department of Astronomy, 1 University Station C1400, Austin, Texas 78712-0259, USA

3 Leiden Observatory, Leiden University, PO Box 9513, 2300 RA Leiden, The Netherlands

${ }^{4}$ Max-Planck-Institut für extraterrestriche Physik. Garching, Germany

5 Herschel Science Centre, European Space Astronomy Centre (ESA), PO Box 78, 28691 Villanueva de la Cañada (Madrid), Spain

6 Department of Astronomy and Space Science, Astrophysical Research Center for the Structure and Evolution of the Cosmos, Sejong University, Seoul 143-747, Republic of Korea

7 Centre for Star and Planet Formation, Natural History Museum of Denmark, University of Copenhagen, Øster Voldgade 5-7, 1350 Copenhagen K., Denmark

${ }^{8}$ Max Planck Institute for Astronomy, Königstuhl 17, 69117 Heidelberg, Germany

9 Instituut voor Sterrenkunde, K.U. Leuven, Celestijnenlaan 200D, 3001 Leuven, Belgium

10 Astronomy Department, University of California, Berkeley, CA 94720, USA

11 Laboratoire d'Astrophysique de Grenoble, CNRS/Université Joseph Fourier (UMR5571) BP 53, 38041 Grenoble Cedex 9, France
12 Department of Astronomy, The University of Michigan, 500 Church Street, Ann Arbor, MI 48109-1042, USA

13 Caltech, Division of Geological \& Planetary Sciences, Mail Code 150-21, Pasadena, CA 91125

14 Naval Research Laboratory, Code 7211, Washington, DC 20375, USA

15 Institute for Astronomy, University of Hawaii at Manoa, Honolulu, HI 96822, USA ; Spitzer Fellow

16 Astronomical Institute "Anton Pannekoek", University of Amsterdam, PO Box 94249, 1090 GE Amsterdam, The Netherlands

17 Department of Astrophysics/IMAPP, Radboud University Nijmegen, PO Box 90106500 GL Nijmegen, The Netherlands

18 Institut für Theoretische Astrophysik, Universitt Heidelberg, AlbertUeberle-Strasse 2, 69120 Heidelberg, Germany

19 University of Vienna, Department of Astronomy, Türkenschanzstr. 17, 1180 Vienna, Austria

20 Department of Astronomy, University of Maryland, College Park, MD 20742, USA

${ }^{21}$ Dpt. de Física Teórica, Fac. de Ciencias, Universidad Autónoma de Madrid, Cantoblanco, 28049 Madrid, Spain

22 SRON Netherlands Institute for Space Research, PO Box 800, 9700 AV, Groningen, The Netherlands

23 National Optical Astronomy Observatory, 950 N. Cherry Ave., Tucson, AZ 85719, USA

24 Postdoctoral Fellow of the Fund for Scientific Research, Flanders, Belgium 